\title{
Advances in HIV laboratory testing
}

\section{Dominic E. Dwyer}

Centre for Infectious Diseases and Microbiology Laboratory Services, Institute of Clinical Pathology and Medical Research, Westmead Hospital

Email:dominic_dwyer@wmi.usyd.edu.au

\begin{abstract}
Laboratory tests are readily available for the diagnosis of HIV infection. These are based on the detection of HIV-specific antibodies and HIV p24 antigen in combination screening assays, followed by confirmation by Western blot. Managing established HIV infection, including the use of antiretroviral drugs, has been facilitated by the use of nucleic acid tests that measure HIV RNA load in plasma or detect mutations associated with drug resistance. Quality assurance programs ensure high-level performance of HIV assays.
\end{abstract}

complexity in antiretroviral therapies and new discoveries relating to HIV pathogenesis mean that patient management remains, logically, in the hands of clinicians with experience in managing HIV infection.

This article introduces the laboratory assays (Box 1) and explains their application in the diagnosis and treatment of HIV.

\section{HIV screening assays}

The keystone of HIV management relies initially on the correct diagnosis of infection, usually by the detection of specific anti-HIV antibodies. Screening programs demand assays that not only identify the presence of HIV antibody reliably, but also have the sensitivity to detect them as early as possible during or after seroconversion. Consent for HIV testing is always needed. The range of items required to be canvassed through pre-test discussion has been relaxed in the Australian Government's National HIV Testing Policy 2006 (available at: http://www.health.sa. gov.au/PEHS/PDF-files/hiv-testing-policy-2006.pdf).

Box 1. HIV-specific laboratory tests used in diagnosis and treatment in NSW

1. Screening assays for HIV antibodies

a. HIV antibody or antigen/antibody combination assays

2. Confirmatory assays

a. Western blot

3. Nucleic acid testing assays to obtain close to $100 \%$ reliability in HIV diagnosis has not changed significantly since the first HIV-specific antibody assays became available in 1985. However, there have been dramatic increases in the sensitivity and specificity of the tests used to diagnose and confirm infection. All Australian laboratories now operate using instrumentbased assays, under quality management systems monitored with quality assurance programs to regularly check their performance.

Nucleic acid testing (NAT) has been introduced to measure HIV levels in plasma, detect HIV in cells, and determine the presence of antiretroviral drug-resistance mutations. More recently, specific assays have become available that help clinicians predict whether antiretroviral-specific adverse effects will occur or whether the viral phenotype predicts response to certain antiretroviral drugs. Increasing
a. Quantitative HIV RNA plasma load
b. HIV DNA on peripheral blood mononuclear cells (PBMC) testing
C. Nucleic acid testing to screen blood donations

4. Guiding therapy
a. Antiretroviral drug-resistance genotyping
b. Coreceptor usage
c. Pharmacogenomic assays

5. Epidemiological and forensic studies
a. HIV subtyping
b. HIV sequencing and phylogenetic analysis
c. Detuned HIV antibody assays

6. Less commonly used assays in Australia
a. Rapid HIV antibody
b. Antiretroviral drug-resistance phenotyping. 
Various generations (denoting significant advancements) in HIV antibody tests, usually based on the enzyme-linked immunosorbent assay (ELISA) format, have evolved over the last two decades, moving from early prototype assays that were neither adequately specific nor sensitive (and time consuming to perform), to the presently used automated, highly sensitive and specific chemiluminescent assays. Commercial assays were refined from 1990 onwards to detect antibodies to HIV-2 or unusual HIV-1 strains. The major recent advance has been the addition of HIV p24 antigen detection to the HIV antibody assays. These 'combination' or 4th generation assays reduce the seroconversion window period to approximately two weeks. Automated combination assays are now used widely in Australia, and have generally replaced screening tests that detect HIV antibody alone, and HIV p24 antigen assays. Interestingly, in the USA and some other countries, combination HIV antibody/antigen assays are not yet routinely available.

\section{HIV confirmatory assays}

The approach to confirmation of a positive anti-HIV screen has not changed significantly in Australia over recent years. Confirmation is still performed by the western blot assay, a test that is technically more difficult and more expensive than the screening ELISA, but when appropriate interpretative criteria are used is still the most specific confirmatory immunoassay. Laboratories that perform HIV screening assays refer reactive samples to reference laboratories for western blot confirmation. In resourcelimited countries where western blot confirmatory testing may be unavailable, sequential use of immunoassays yields reliable positive results.

Rapid (point-of-care) assays that detect HIV-specific antibodies require less technical expertise to perform and are more commonly used in resource-limited settings. They have lower sensitivity and specificity compared with standard assays. They are not used in Australia except in urgent clinical situations such as postexposure prophylaxis management or pre-transplantation screening. Retesting with standard immunoassays is required by the National HIV Testing Policy (available at: http://www.health.sa.gov. au/PEHS/PDF-files/hiv-testing-policy-2006.pdf). Assays are available (but rarely used in Australia) that allow HIV antibodies to be identified in saliva or other body fluids, and are designed to allow people to self-sample. For surveillance purposes, ELISA HIV-antibody tests can be 'detuned' to detect more recent infection.

HIV isolation from blood or other clinical samples is definitive evidence of infection. However, it takes between 2 and 4 weeks, and is diagnostically insensitive. It was useful before the development of NAT in detecting infection in babies born to mothers with HIV infection. It is still used for research purposes, and sometimes as part of epidemiological investigations.

\section{Nucleic acid testing (NAT) in HIV}

The development of assays for reliable quantitative NAT for measuring levels of HIV RNA (viral load) in plasma or other body fluids has revolutionised clinical management. Viral load assays, along with CD4+ T lymphocyte cell counts, provide crucial prognostic information to clinicians and patients. Plasma NAT assays are used to monitor the efficacy of antiretroviral drugs, aimed at maintaining viral loads below detectable levels. Along with rising CD4+ $\mathrm{T}$ cell counts, low plasma HIV loads demonstrate successful antiretroviral therapy. A significant rise in plasma HIV load suggests treatment failure, but does not determine whether the cause is antiretroviral drug resistance, or problems with treatment compliance. Qualitative NAT is used for screening of the blood supply in Australia.

NAT to detect HIV DNA in peripheral blood mononuclear cells is the first test to become positive (at approximately one week) in HIV seroconversion. It is also in the test of choice in babies born to mothers with HIV infection. HIV DNA testing is highly sensitive and specific, and, along with the commercial viral load assays, has improved the ability to detect and quantify unusual HIV subtypes.

\section{Guiding therapy \\ Genotypic HIV drug resistance testing and subtyping}

NAT is also used to determine when specific mutations for antiretroviral drug resistance are present in a person's circulating HIV. Genotyping is performed by sequencing the relevant antiretroviral drug target sequences (e.g. reverse transcriptase or protease) in the circulating HIV genome. Sequences are then compared with large international HIV sequence databases and specific mutations are defined and reported to the clinician with comments about whether these mutations are consistent with clinical resistance. As new drug classes are developed, genotyping has expanded to include new antiretroviral targets e.g. integrase or envelope gp41.

The analysis of reverse transcriptase and protease sequences for antiretroviral drug resistance also generates a HIV subtyping result. This has replaced older subtyping methods. HIV subtyping serves epidemiological purposes, determining if uncommon HIV strains are emerging.

\section{Phenotypic HIV drug-resistance testing}

A virtual phenotype can be generated by comparing a genotype with databases that have information on specific sequences and their associated phenotypes. True phenotypic 
testing is expensive and is currently only available in Australia in clinical trials. Phenotyping generally involves the amplification of reverse transcriptase or protease, insertion of the sequences into a plasmid vector, then culturing a hybrid virus containing that vector in comparison with wild-type HIV. Phenotypic resistance assays are most valuable in the management of highly antiretroviral therapyresistant patients.

\section{Other HIV assays used in antiretroviral therapy}

The development of drugs that target HIV coreceptors has led to the development of assays that measure tropism of a person's virus for either (or both) coreceptors, CCR5 and CXCR4. CCR5 antagonists are only effective if the virus dominantly uses CCR5 as its coreceptor. These assays are needed only when coreceptor antagonists are planned as part of combination antiretroviral therapy. A NAT-based pharmacogenomic assay that detects the HLA-B5701 allele is now routinely used in anyone starting abacavir to avoid severe hypersensitivity.

\section{HIV epidemiological and forensic investigations}

HIV sequencing can be used in epidemiological investigations, for example in detecting nosocomial transmission or unusual HIV clusters. These involve sequencing parts of, or the entire, HIV genome, followed by phylogenetic analysis to determine HIV strain relatedness. Such investigations are carried out in conjunction with public health or forensic specialists.

\section{Laboratory quality assurance for HIV testing}

Another key to the reliability of HIV testing is the required participation by laboratories in ongoing quality assurance programs. The obligatory programs are administered by the National Serology Reference Laboratory which also evaluates commercial assays for the Therapeutics Goods Administration. Participation in the National Serology Reference Laboratory's quality assurance programs does not preclude participation in the quality assurance programs of the Royal College of Pathologists of Australasia or international bodies. Quality assurance programs have a fundamental role in ensuring high-level performance of HIV assays.

\section{Conclusion}

The detection of early HIV infection has improved with the application of combination HIV antibody/antigen assays. Reactive screening ELISA tests still require confirmation by western blot and/or other assays. The development of HIV plasma load and other laboratory tests that detect antiretroviral drug resistance have improved patient and antiretroviral treatment management. Quality management and quality assurance programs are pivotal in assuring the ongoing high reliability of HIV assays in Australia. 